\title{
Viscosity of Unimodal and Bimodal Alumina/Epoxy Mixtures
}

\author{
Noriyuki Hayashi* Member \\ Shin-ichiro Tsuru ${ }^{* *}$ Member \\ Wibowo Sulistio ${ }^{*}$ Member \\ Yasushi Sakamoto $^{* * *}$ Non-member Masanori Hara ${ }^{* * * *}$ Member
}

\begin{abstract}
Experimental results on characteristics of viscosity $\eta$ of unimodal and bimodal alumina/epoxy mixtures are shown and discussed based on theoretical consideration. Spherical alumina particles with nominal diameters of $35 \mu \mathrm{m}$ and $80 \mu \mathrm{m}$, both of which had narrow dispersion of diameter distribution, were used as fine and coarse particles, respectively. $\eta$ of the unimodal mixture increases remarkably with a volume packing fraction of particle $\nu_{p}$, and that Brinkman equation can be applied to estimate $\eta$ from $\nu_{p}$. On the other hand, $\eta$ of the bimodal mixture depends not only on $\nu_{p}$, but also on the ratio of coarse particles to fine ones. For larger $\nu_{p}, \eta$ of the bimodal mixture becomes smaller than that of the unimodal mixture. It is shown that an approximation procedure of $\eta$ yields reasonable estimation for smaller $\nu_{p}$, and underestimation for larger $\nu_{p}$.
\end{abstract}

Keywords: functionally graded material, alumina/epoxy mixture, viscosity, bimodal system, unimodal system

\section{Introduction}

The authors are using a centrifugal method to fabricate functionally graded materials with large size from alumina-particles/epoxy-resin mixtures ${ }^{(1)}$. During the centrifugal process, drag force is one of the major forces experienced by the particles, and hence, it dominates the movement of the particles. It directly depends on the coefficiency of viscosity of the mixture, $\eta$, and consequently, precise information of $\eta$ is indispensable for both the fabrication and simulation process ${ }^{(1)}{ }^{(2)}$.

To evaluate effects of the amount and size of particles involved in the mixture on $\eta, \eta$ of unimodal and bimodal alumina/epoxy mixtures were measured under various conditions. Experimental results on the characteristics of $\eta$ are demonstrated and theoretically discussed.

\section{Materials and Experimental Method}

Spherical alumina particles with nominal diameters of $35 \mu \mathrm{m}$ and $80 \mu \mathrm{m}$ were used as fine and coarse particles. Mixtures involving either particle can be regarded as a unimodal system, because this particle had narrow dispersion of diameter distribution ${ }^{(3)}$. Meanwhile, the bimodal mixtures involving both particles represent mixtures with suspensions such as a pulverised powder that has a variety of diameters ${ }^{(1)(3)}$.

For the unimodal mixture, when particles by the volume $V_{p}$ were mixed with an epoxy resin by the volume $V_{e}$, the volume packing fraction of particles is given by $\nu_{p}=V_{p} /\left(V_{p}+V_{e}\right)$. On the other hand, for the bimodal mixture, both the fine and coarse particles by

* ASEM, Faculty of Engineering Sciences, Kyushu University 6-1, Kasuga Kohen, Kasuga 816-8580

** Research Lab., Kyushu Electric Co., Inc. 2-1-47 Shiobaru, Minami-ku, Fukuoka 815-8520

*** Nishi Nippon Electric Wire \& Cable Co., Ltd. Kasugaura, Oita 870-0011

**** EES, Faculty of Information Sciences and Electrical Engineering, Kyushu University

6-10-1, Hakozaki, Higashi-ku, Fukuoka 816-8580 the volume of $V_{f}$ and $V_{c}$, respectively, were mixed with an epoxy resin. In this case, two kinds of the volume fraction of particles can be defined: one is ratio of all particles to the mixture $\nu_{p}=\left(V_{f}+V_{c}\right) /\left(V_{f}+V_{c}+V_{e}\right)$, and the other is ratio of the coarse particles to all particles $\nu_{\text {coarse }}=V_{c} /\left(V_{f}+V_{c}\right)$.

After a given amount of fine and coarse alumina particles was dispersed in an epoxy resin, the alumina/epoxy mixture was de-gassed in a vacuum chamber. Then, $\eta$ of the mixtures was measured by the viscosity meter when temperature of the mixture became $26 \pm 0.5{ }^{\circ} \mathrm{C}$.

\section{Experimental Results and Discussions}

3.1 Viscosity of Unimodal Mixtures $\nu_{p}$ varied from 0 to $45 \%$ for both alumina particles.

Fig. 1 shows dependency of $\eta$ on $\nu_{p}$. Two symbols of - and $\circ$ in Fig. 1 show experimental values of $\eta$ obtained for the fine and coarse particles, respectively. Fig. 1 indicates that $\eta$ increases remarkably with $\nu_{p}$ for both particles. It is also clear that $\eta$ of the fine particles is slightly larger than that of the coarse ones, and the difference in $\eta$ becomes notable as $\nu_{p}$ increases.

It is known that $\eta$ of a unimodal mixture is gener-

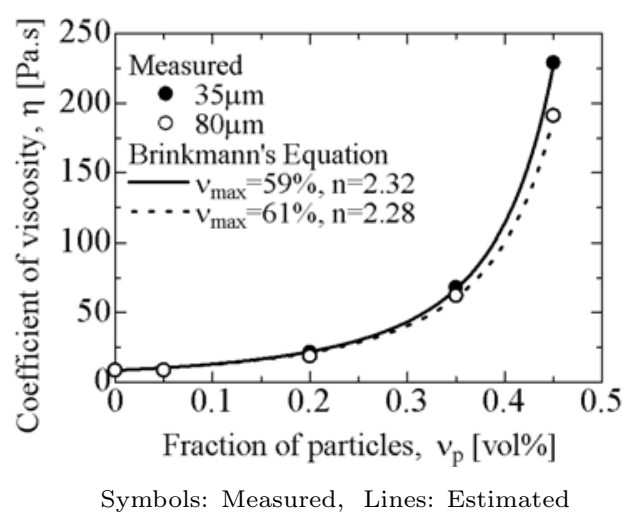

Fig. 1. $\quad \eta$ of unimodal mixtures as a function of $\nu_{p}$ 
ally given as a function of $\nu_{p}$ by the following equation proposed by Brinkman ${ }^{(4)}$ :

$$
\eta\left(\nu_{p}\right)=\eta_{0} /\left(1-\frac{\nu_{p}}{\nu_{\max }}\right)^{n}
$$

where $\eta_{0}$ is the coefficient of viscosity of the epoxy resin, $\nu_{\max }$ is the maximum packing fraction obtainable for the mixtures under consideration, and $n$ is a constant.

Values of $\nu_{\max }$ and $n$ in Eq. 1 were calculated to fit the measured values shown in Fig. 1. $\nu_{\max }=0.59$ and $n=2.32$ are obtained for the fine particles, while $\nu_{\max }=0.61$ and $n=2.28$ are obtained for the coarse particles.

These values of $\nu_{\max }$ and $n$ along with $\eta_{0}=8.5 \mathrm{~Pa} \cdot \mathrm{s}$ were used to calculate $\eta$ for various $\nu_{p}$, and profiles of $\eta$ thus obtained are plotted by solid and broken lines for the fine and coarse particles, respectively, in Fig. 1. Comparison between the measured and estimated values shown in Fig. 1 demonstrates that the Brinkman equation expressed in Eq. 1 can be applied to estimate $\eta$ of the unimodal alumina/epoxy mixture from $\nu_{p}$ with reasonable precision.

3.2 Viscosity of Bimodal Mixtures $\quad \nu_{p}$ varied between 0 to $45 \%$, and $\nu_{\text {coarse }}$ varied between 0 to $100 \%$ for each $\nu_{p}$. It is noted that the bimodal mixtures with $\nu_{\text {coarse }}=0 \%$ and $100 \%$ correspond to the unimodal mixtures with the fine and coarse particles only, respectively.

Symbols in Fig. 2 show measured values of $\eta$ obtained for various $\nu_{p}$ and $\nu_{\text {coarse }}$. It is obvious from Fig. 2 that $\eta$ of the bimodal mixture depends not only on $\nu_{p}$, but also on $\nu_{\text {coarse }} . \eta$ is almost independent of $\nu_{\text {coarse }}$ for smaller $\nu_{p}$ up to $20 \%$. Meanwhile, for larger $\nu_{p}$ more than $35 \%$, $\eta$ depends on $\nu_{\text {coarse }}$, and $\eta$ of the bimodal mixture becomes smaller than that of the unimodal mixture (i.e. $\nu_{\text {coarse }}=0 \%$ or $100 \%$ ), and reaches a minimum value at a certain $\nu_{\text {coarse }}$ of $\nu_{c, \text { min }}$ around $60 \%$.

Based on the approximation procedure of $\eta$ of the bimodal mixture, it can be predicted by using $\eta$ of the unimodal one, and is given by the following expression ${ }^{(5)}$.

$$
\eta\left(\nu_{p}, \nu_{\text {coarse }}\right)=\eta_{0} \cdot \frac{\eta_{f}\left(\nu_{f}\right)}{\eta_{0}} \cdot \frac{\eta_{c}\left(\nu_{c}\right)}{\eta_{0}} \cdots \ldots \ldots
$$

Here, $\eta_{f}\left(\nu_{f}\right)$ and $\eta_{c}\left(\nu_{c}\right)$ are $\eta$ of the unimodal mixtures involving the fine and coarse particles, respectively,

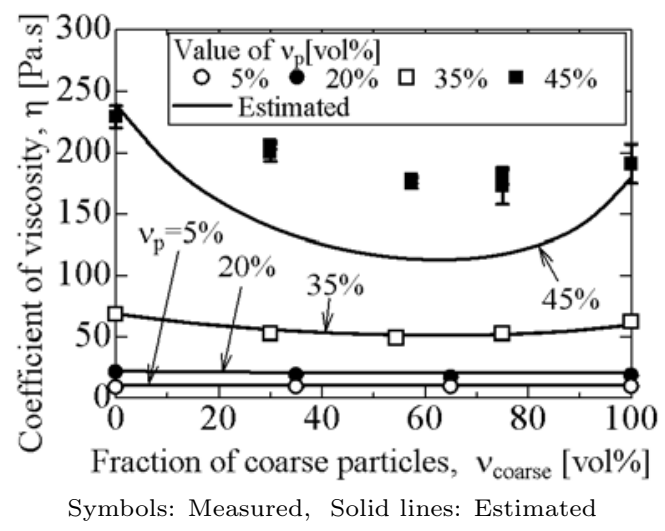

Fig. 2. $\quad \eta$ of bimodal mixtures as a function of $\nu_{\text {coarse }}$ for various $\nu_{p}$ and are estimated by using Eq. 1 in advance, where $\nu_{f}=V_{f} /\left(V_{e}+V_{f}\right)$ and $\nu_{c}=V_{c} /\left(V_{e}+V_{f}+V_{c}\right)$.

Furthermore, $\nu_{c, \text { min }}$, at which $\eta$ becomes the smallest, is estimated by the following expression that includes $\nu_{p}$ and $V_{e}$ as given values ${ }^{(5)}$.

$$
\nu_{c, \min }=V_{c}^{\prime} /\left(V_{f}^{\prime}+V_{c}^{\prime}\right) \ldots \ldots \ldots \ldots \ldots \ldots \ldots
$$

where, $V_{f}^{\prime}=\nu_{p}^{\prime} V_{e} /\left(1-\nu_{p}^{\prime}\right), V_{c}^{\prime}=\nu_{p}^{\prime}\left(V_{e}+V_{f}^{\prime}\right) /\left(1-\nu_{p}^{\prime}\right)$, and $\nu_{p}^{\prime}=1-\sqrt{1-\nu_{p}}$.

For the bimodal mixture under consideration, dependency of $\eta$ on $\nu_{\text {coarse }}$ were calculated using Eq. 2 for $\nu_{p}=5,20,35,45 \%$, and the results are plotted by solid lines in Fig. 2. Moreover, $\nu_{c, \min }=51,53,55,57 \%$ for $\nu_{p}=5,20,35,45 \%$, respectively, were obtained by Eq. 3 .

It is obvious from Fig. 2 that the approximated results by Eq. 2 quantitatively express dependency of $\eta$ against $\nu_{\text {coarse }}$, and that, in particular, the reduction of $\eta$ for the bimodal mixtures clearly appears at larger $\nu_{p}$. The approximation procedure yields reasonable estimation of $\eta$ for $\nu_{p}$ smaller than $35 \%$, while it underestimates $\eta$ for $\nu_{p}$ larger than $45 \%$. Meanwhile, $\nu_{c, \min }$ is estimated by Eq. 3 with reasonable precision regardless of $\nu_{p}$.

\section{Conclusions}

Experimental results on characteristics of $\eta$ of unimodal and bimodal alumina/epoxy mixtures are shown and discussed based on the theoretical consideration.

$\eta$ of the unimodal mixtureincreases remarkably with $\nu_{p}$, and Brinkman equation can be applied to estimate $\eta$ from $\nu_{p}$. On the other hand, $\eta$ of the bimodal mixture depends not only on $\nu_{p}$, but also on $\nu_{\text {coarse }}$. For larger $\nu_{p}, \eta$ of the bimodal mixture becomes smaller than of the unimodal mixture, and reaches a minimum value at a certain $\nu_{\text {coarse }}$. An approximation procedure proposed by Farris gives reasonable estimation of $\eta$ for smaller $\nu_{p}$, while it underestimates $\eta$ as $\nu_{p}$ increases.

\section{Acknowledgment}

This work is partly supported by Grant-in-aids for Scientific Research (C)(2) \#16560244, and Joint research project on FGM between Kyushu University, Kyushu Electric Power Co., Inc., and Nishi Nippon Wire \& Cable Co., Inc.

(Manuscript received Sep. 21, 2004)

\section{References}

(1) N. Hayashi, S.I. Tsuru, T. Onoda, Y. Sakamoto, and M. Hara: "Fabrication of Functionally Graded Epoxy Resin with Alumina Fillers and Its Simulation by Using a Centrifugal Method", IEEJ Trans. FM, Vol. 124, No. 7, pp. 598-606 (2004)

(2) Y. Watanabe, A. Kawamoto, and K. Matsuda: "Control of Composition Gradient in a Metal-Ceramic Functionally Graded Material Manufactured by the Centrifugal Method", Composites Part A, No. 29A, pp. 595-601 (1998)

(3) N. Hayashi, T. Onoda, Y. Sakamoto, S.I. Tsuru, T. Kawabe, and M. Hara: "Fabrication of Permittivity Graded Epoxy Resin with Non-Uniform Dispersion of Alumina Fillers by Centrifugal Procedure", Materials Science Forum, (in press)

(4) H.C. Brinkman: "The Viscosity of Concentrated Suspensions and Solutions", J. Chem. Phys, Vol. 20, No. 4, p. 571 (1952)

(5) R.J. Farris: "Prediction of the viscosity of Multimodal Suspensions from Unimodal Viscosity Data", Trans. Soc. Rheology, Vol. 12:2, pp. 281-301 (1968) 